\title{
Guinea-worm Arthritis of Knee Joint
}

Standard textbooks on parasitology do not mention the exact cause of arthritis in guinea-worm infection. Manson-Bahr (1954) reported that Botreau-Roussel and Huard described a specific non-bacterial arthritis, especially of the knee joint, associated with the presence of a guinea-worm in the vicinity. Manson-Bahr, in discussing sequelae of guinea-worm infection, stated that direct spread of secondary infection to the synovial membrane gives rise to arthritis which may lead to fibrous ankylosis. Faust and Russell (1957) mentioned that the sequelae of guinea-worm infection include synovitis, arthritis, and ankylosis but did not describe the mode of pathogenesis. Chatterjee (1952) stated that owing to secondary infection superimposed on a guinea-worm tract a neighbouring joint may be involved. Middlemiss (1961) explained involvement of the knee joint in guinea-worm infection as being due to a reactionary effusion when the worm dies in close proximity to a joint. Connor (1922-3) believed that joints are involved from the presence of calcified worms outside the joint. Adams and Maegraith (1966) stated that joint involvement occasionally occurred without secondary infection, but they failed to mention how the joint gets involved. Onabamiro (1958) thought that a secondary infection of a sterile abscess of a guinea-worm occurring near a joint produces septic arthritis.

In our experience in an area where guinea-worm infection is still very common the involvement of the knee joint is due to the entry into it of the worm and liberation of the larvae. In one summer we came across four cases of acute arthritis of the knee joint in which we were able to show the adult worm inside the joint.

\section{Four Cases}

Clinical Findings.-All four patients were adult manual labourers who came from endemic areas of guinea-worm infection and had a previous history of guinea-worm infection. They had had rash and itching all over the body three to four days before the onset of synovitis. The first case came three days after the onset of swelling of the joint, and the others after 15,4 , and 2 days respectively. The first patient felt the movement of the worm subcutaneously near the suprapatellar region three days before the onset of synovitis. In all cases the onset was sudden and gross limitation of movement was present. Movements were painful in all cases and the infected leg could not be used. In all cases the temperature was raised and the joint was swollen, tender, and warm, and contained fluid. Radiological examination of the knee region failed to show any calcified worm.

Synovial Fluid.-The aspirated fluid was yellowish and turbid, and contained flakes of soft yellowish material. Microscopical examination showed live guinea-worm larvae in one case and dead ones in the other three. The cells in the exudate were adherent to the larvae. Bacteriological examination of the fuid in all cases failed to show any organisms. Cellular exudate consisied of monocytes, plasma cells, polymorphonuclear cells, and a few eosinophils. There was no evidence of fibrin in any of the cases.

\footnotetext{
- Professor of Pathology.

t Professor of Orthopaedics.

Kurnool Medical College, Kurnool (A.P.), South India.
}

Operation.-In all four cases the joint was exposed through a shurt anteromedial or anterolateral incision. Appearances at operation were similar in all cases. Fluid was turbid and yellow. Synovial membrane was thickened to 2 to $3 \mathrm{~mm}$. by oedema and congestion and thrown into redundant folds; it was red in colour and covered by exudate. The suprapatellar pouch was mainly involved. The worm was seen on the femoral surface of the suprapatellar pouch, and part of it protruded into the joint cavity (see Fig.). In two cases the worm was lying coiled and free and could be removed easily. In the other two cases part of the synovial membrane had to be excised to remove the worm, as it was partly embedded in the membrane. Irrigation of the joint cavity with normal saline revealed a normal shiny surface of the synovial membrane and the wound was closed in layers. Compression bandage and plaster-ofParis cast were applied. On the tenth day sutures were removed. In all four cases effusion subsided, and the patients were allowed to walk with a compression bandage. One month later the thickening of the synovial membrane had completely disappeared. The range of movement regained was more than $90^{\circ}$. All the patients went back to their hard work satisfied with the result.

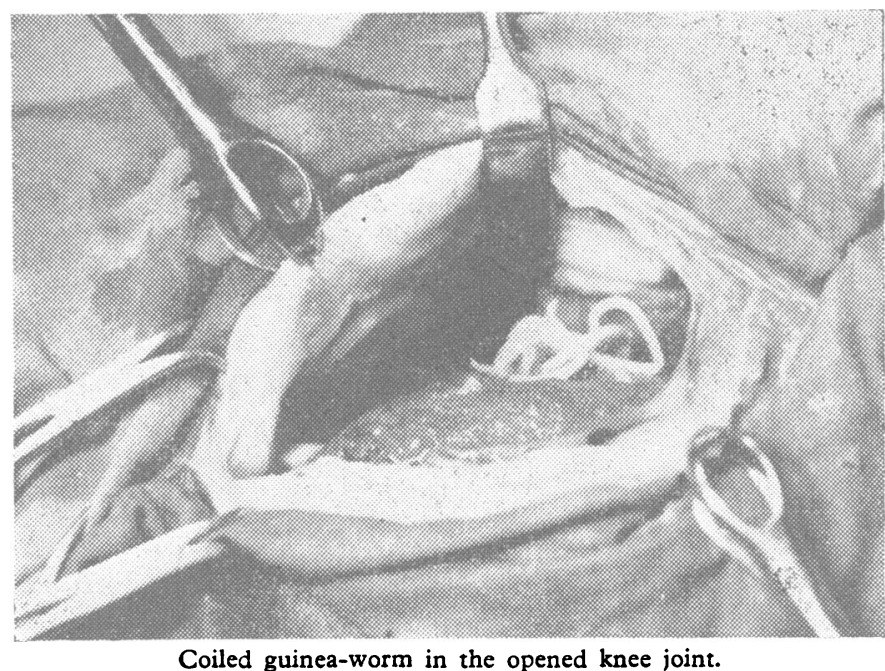

Pathology.-In the early stages synovial membrane showed congestion, oedema, and infiltration with plasma cells, and in the later stages increased amounts of fibrous tissue. In all four cases the worm was entire and intact. It did not show any rupture or break of the body. The worm in the fourth case was alive and in the other cases dead.

\section{Comment}

We could find only one other report of a guinea-worm in the knee joint (Gandhi, 1962). The route of entry of the worm into the joint is probably via the suprapatellar pouch, but no definite proof of this except for the fact that the worm was seen there. Very little is known about the development and migration of the worm from the stage of infective larvae to the adult stage. The entry of the worm into the knee joint cavity to discharge its larvae is probably just an abnormal mode of 
discharging the larvae by a mature worm. Guinea-worms are known to discharge larvae in abnormal sites such as the extradural space (Reddy and Valli, 1967). The arthritis is not due to secondary infection, as in all the cases the culture was sterile. It is due to the products of parturition of the worm discharged into the joint. It is not due to the breaking up and crushing of the worm between the opposing bones during walking, for in every case the worm was entire without any break. In the last case operation was carried out on the third day after the onset of pain and swelling, and the worm was alive and moving. This again shows that it is the discharge of the larvae in an abnormal site which is responsible for the synovitis.

Gandhi (1962) treated his case by rest and antibiotics only after the chance removal of the worm (which got caught in the aspirating needle) and aspiration of the fluid. No mention of seeing the larvae was made in the report nor was a follow-up noted.

As a rule surgical procedures are carried out after the active stage of synovitis has been arrested, and conservative treatment should be given an adequate trial before surgery is undertaken. Conservative treatment in several cases of acute synovitis of the knee with a history of guinea-worm infection during the past few years was not successful. Entry of the worm into the joint cavity and liberation of its products of conception, giving rise to acute synovitis, was not thought of. So repeated aspirations with instillation of penicillin were given. Culture examination was always sterile and no definite diagnosis was made. Simple microscopical examination of the fluid was not made, as the finding of larvae in the fluid was not expected. Many cases ended up with chronic synovitis and involvement of cartilaginous and osseous portions of the joint, and were mistaken for gonococcal or tuberculous arthritis. Fluid in the joint with chronic proliferation of the synovial membrane persisted for months. Surgery was undertaken late in these cases in the form of synovectomy or arthrodesis, with consequent partial or complete stiffness of the knee after months of suffering.

Routine microscopy of the joint fluid in cases of acute synovitis disclosed larvae, and since larvae could not be seen with- out the presence of the adult worm immediate removal of the worm and its extruded products from the joint was thought to be imperative. So arthrotomy in the acute stages was felt to be necessary for the removal of the offending worm and products, with irrigation of the joint cavity.

In our limited experience of this not so unusual but definitely unsuspected lesion we found that if the patient reported early, when the larvae could be seen in the fluid, the diagnosis was easy. In such cases arthrotomy for removal of the worm should be done. Removal of the worm and cleaning of the joint cavity was sufficient to restore a joint to normal use.

\section{Summary}

The pathogenesis of arthritis of the knee joint in dracontiasis is not established. In four cases of acute arthritis of the knee joint guinea-worm larvae were found in the aspirated fluid and at arthrotomy the adult worm was seen in the joint. In all cases the worm was entire and in one case it was alive. The aspirated fluid was sterile on culture. This shows that the arthritis was due to the discharge of larvae by the worm in an ectopic site. Removal of the worm and cleaning of the joint cavity restored the joint to normal. If this is not done chronic synovitis and possible fibrous ankylosis will ensue.

\section{REFERENCES}

Adams, A. R. D., and Maegraith, B. G. (1966). Clinical Tropical Disease, 4 th ed. Oxford.

Chatterjee, K. D. (1952). Human Parasites and Parasitic Diseases. Calcutta.

Connor, F. P. (1922 -3). Brit. F. Surg., 10, 253.

Faust, E. C., and Russell, P. F. (editors) (1957). Craig and Faust's Clinical Parasitology, 6th ed. Philadelphia.

Gandhi, N. J. (1962). Brit. med. F., 1, 1206.

Gandhi, N. J. (1962). Brit. med. Manson's Tropical Diseases, 14th ed. London.

Middlemiss, H. (1961). Tropical Radiology. London.

Onabamiro, S. D. (1958). In Diseases of Children in the Subtropics and Tropics, edited by H. C. Trowell and D. B. Jelliffe, p. 815. London. Reddy, C. R. R. M., and Valli, V. V. (1967). Amer. F. trop. Med. Hyg., 16, 23.

\section{K. F. FAIRLEY,* M.D., M.R.C.P., F.R.A.C.P. ; PRISCILLA KINCAID-SMITH,† M.B., B.SC., M.R.C.P., F.R.A.C.P., D.C.P.}

Brit. med. F., 1968, 1, 156-157

The concept that the primary lesion in analgesic nephropathy is in the medulla and that the cortical changes of so-called "chronic interstitial nephritis" are secondary (Kincaid-Smith, 1967) has been opposed on the ground that small kidneys may show an apparently normal caliceal system (Krikler, 1967).

We have encountered many patients in whom calices were well cupped and careful scrutiny of whom failed to reveal diagnostic radiological evidence of papillary necrosis in spite of unequivocal pathological evidence of papillary necrosis in biopsy, necropsy, or voided material. Thus we concluded that the renal papillary necrosis cannot be excluded on radiological grounds (Dawborn et al., 1966).

To re-emphasize this point we illustrate it with the following case.

\section{Case Report}

A 41-year-old woman was admitted to the hospital complaining of breathlessness. She had taken 6-10 analgesic tablets containing aspirin, phenacetin, and caffeine daily for 20 years. She was in

* Outpatient Physician, Royal Melbourne Hospital.

† First Assistant in Medicine, University of Melbourne. Physician-inCharge, Medical Renal Unit, Royal Melbourne Hospital, Melbourne, Australia. congestive heart failure, and had a blood pressure of 180/140, a blood urea of $124 \mathrm{mg} . / 100 \mathrm{ml}$, and a haemoglobin of $50 \%$.

An infusion intravenous pyelogram showed very little excretion of contrast medium and a retrograde pyelogram showed a normal caliceal system with no evidence of papillary necrosis (Fig. 1), but the kidneys were small $(10 \mathrm{~cm}$. in length). A renal biopsy revealed
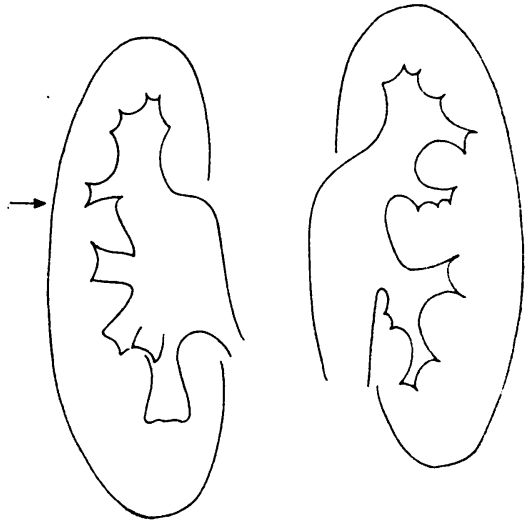

FIG. 1.-Tracing of the retrograde pyelogram. The arrow indicates the calix shown in Fig. 2. 